\title{
Fixed cutaneous sporotrichosis
}

\author{
Patricia Chang ${ }^{1}$, Mónica Vanesa Vásquez Acajabón ${ }^{1}$, Heidi Logemann², Gylari Calderón Pacheco ${ }^{1}$ \\ ${ }^{1}$ Department of Dermatology, Hospital General de Enfermedades IGSS and Hospital Ángeles, Guatemala, ${ }^{2}$ Laboratorio \\ Clinico Micologico Candelaria, Guatemala \\ Corresponding author: Dr. Patricia Chang, E-mail: pchang2622@gmail.com
}

\begin{abstract}
The sporotrichosis is the most common sub cutaneous mycosis in our country and the lymphocutaneous type is the most frequent clinical presentation. Case report: a case of cutaneous fixed sporotrichosis has been reported in a woman of 46 years old with good response to itraconazole. Conclusion: the fixed cutaneous type is less well recognized. In this type, the lesions are restricted to the site of inoculation without any lymphatic spread. Important clues from the clinical history, such as travel and occupation, can help to raise the suspicion of this infection in the differential diagnosis. tissue culture are necessary to confirm the mycological diagnosis.
\end{abstract}

Key words: Cutaneous fixed sporotrichosis, Sporothrix schenckii, itraconazole

\section{INTRODUCTION}

Sporotrichosis is a subcutaneous fungal infection first described by Benjamin Schenks in 1898. It is caused by the dimorphic fungi complex Sporotrhix [1,2] as the result of traumatic inoculations with contaminated material, usually spindles and thorns. Thus, this disease is popularly known as "rose gardener's disease", though it may also be transmitted by cats $[1,2]$. It is considered as a mycosis of global distribution, but its observed with more frequency in tropical countries, having higher incidence in Latin America [1,3]. The clinical types of sporotrichosis are lymphocutaneous sporotrichosis, fixed cutaneous and extracutaneous. Moreover, the heterogeneous morphology of lesions often makes the clinical diagnosis difficult, thereby leading to delayed treatment [1-3].

\section{CASE REPORT}

Female patient, 46 years, who consulted a private doctor due to her dermatological condition in her face region was sent for diagnosis. Upon examination, a dermatosis was found on the left cheek, a plaque with ulcerations on an erythematous base (Fig. 1). The rest of the physical exam was within normal limits.
Familiar and personal history was found negative.

The patient indicated that the disease began 3 months ago with a small asymptomatic "pimple" on the left cheek, discharging pus, and afterwards, slowly increased and ulcerated. She received different antibiotics and non-specific creams with no effective results Three clinical diagnosis were made based on the clinical data: inflammatory, neoplasic or infectious disease. A biopsy was performed, and the histologic sections of skin stained with HE, showing epidermis with hyperkeratosis and irregular acanthosis. From the papillary dermis to the deep reticular dermis, a diffuse granulomatosis infiltrate present at cutting surface was observed (Fig. 2).

At a higher magnification, the infiltrate was formed by granulomas with suppurative center, lymphocytes, histiocytes, plasma cells and abundant giant cells of Langhans type. Moreover, large number of yeast was observed presenting an elongated and oval shape, which was evident on PAS staining (Fig. 3). Afterwards the clinical diagnoses of fixed cutaneous sporotrichosis was performed. Upon re-interrogation, the patient revealed receiving a blow on her left cheek with a

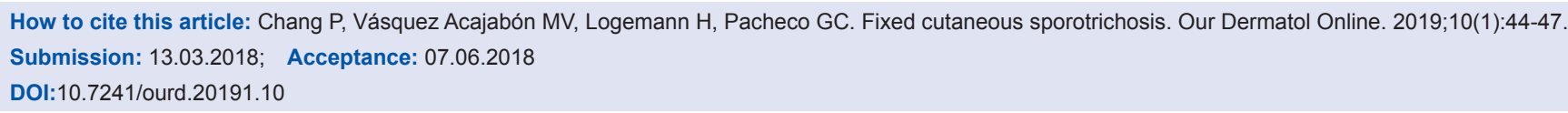




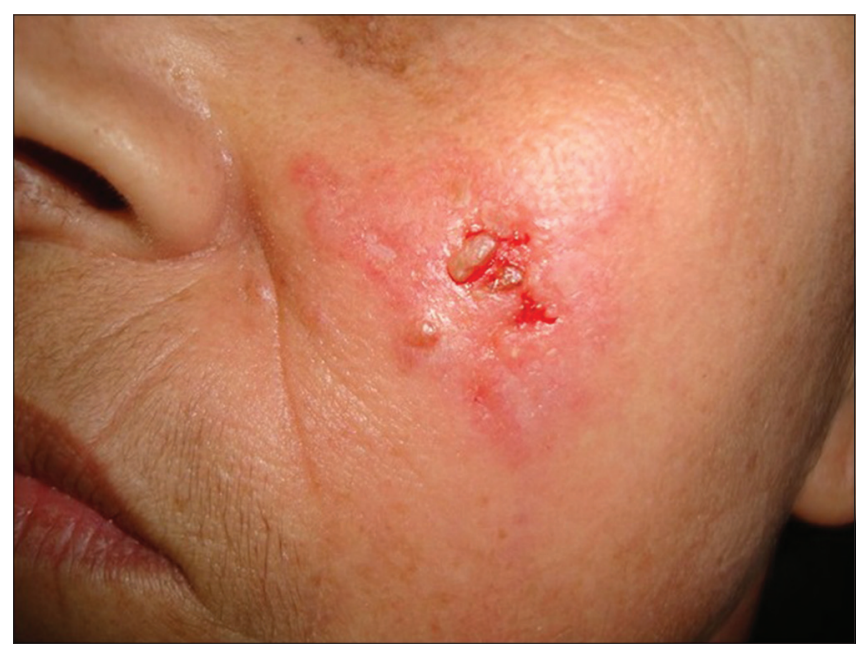

Figure 1: Ulcerations on a well-defined erythematous plaque of $2 \times 2 \mathrm{~cm}$.

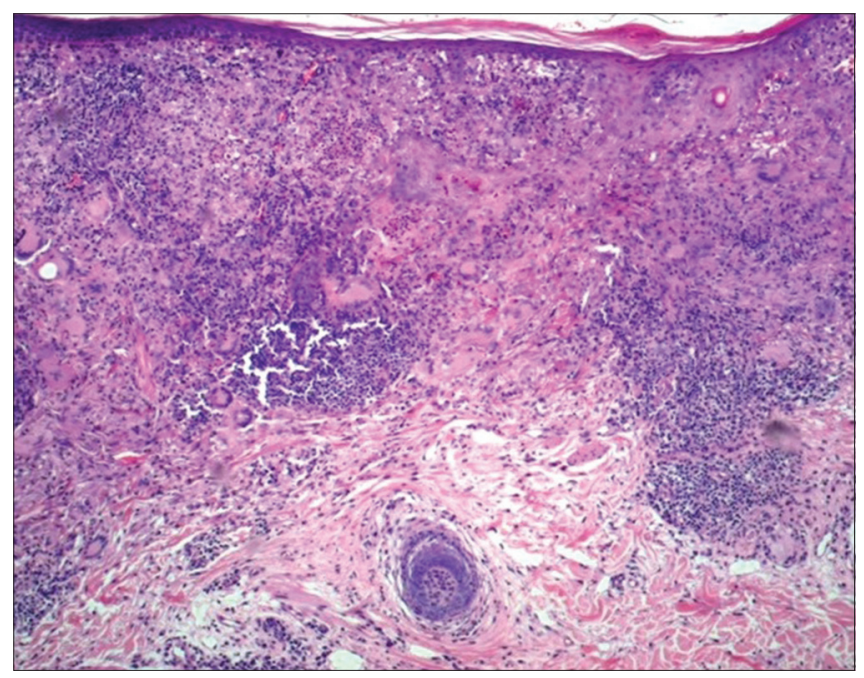

Figure 2: Epidermis with hyperkeratosis, irregular acanthosis. From the papillary dermis to the deep reticular dermis, a diffuse granulomatosis infiltrate.

can of gaseous water about 9 months ago. A fungus cultivation was carried in Sabouraud's glucose agar, surface growth was brown, glabrous and yeast like, with reverse also brown (Fig. 4). The microscopy confirmed Sporothrix schenkii (Fig. 5), the microscopic morphology of the colony was septate, branching hyphae and cluster spores. Moreover, conidia are in a flowerette arrangement at the single conidiophores (Fig. 6). The final diagnosis of the patient was fixed cutaneous sporotrichosis and itraconazole $200 \mathrm{mg}$ daily was prescribed for 6 weeks, resulting in clinical cured (Fig. 7).

Prior to the study, patient gave written consent to the examination and biopsy after having been informed about the procedure.

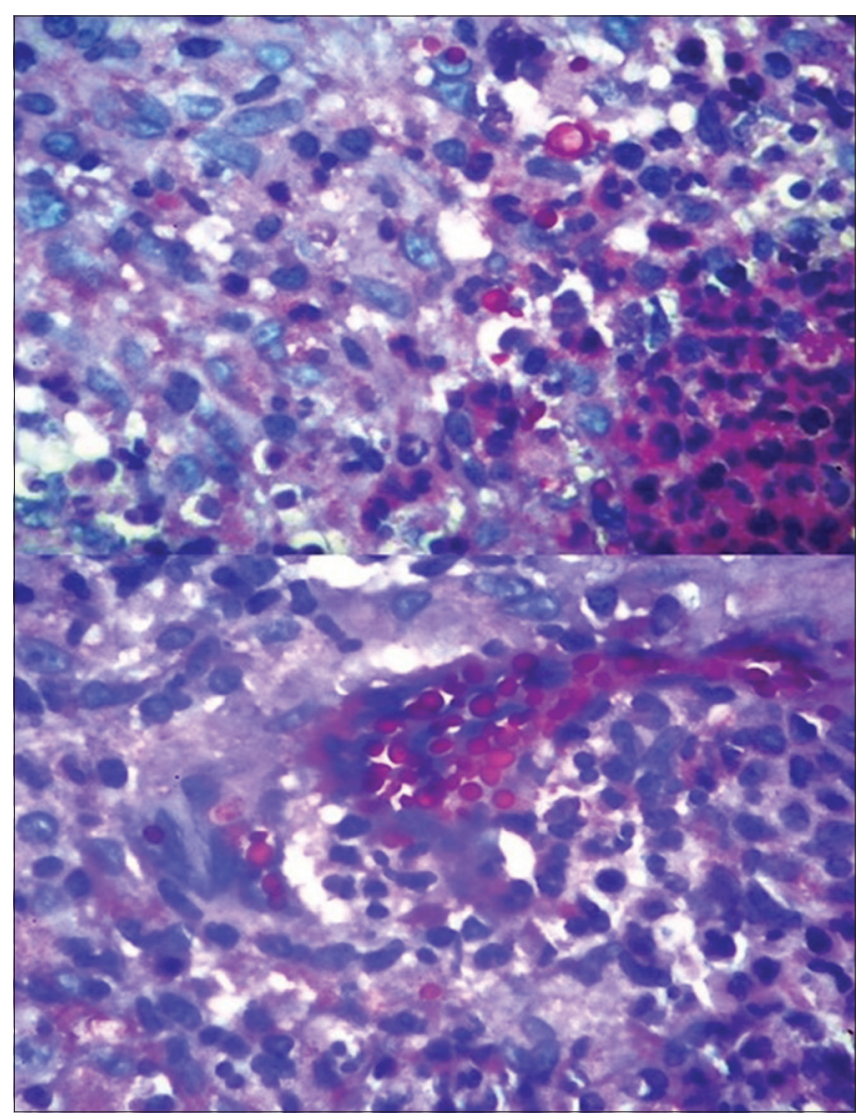

Figure 3: Close up the infiltrate were formed by granulomas with suppurative center, lymphocytes, histiocytes, plasma cells and abundant Langhans type giant cells, a large number of yeast are observed in elongated and oval form, which were evident on PAS staining.

\section{DISCUSSION}

Sporotrichosis, or rose gardener's disease [1], was first described in 1898 by Benjamin Schenk at Johns Hopkins Hospital and was named by Hektoen and Perkins in 1900 $[1,2]$. The disease is a polymorphic subacute or chronic subcutaneous mycosis. Sporotrichosis is dispersed worldwide and more prevalent in tropical and subtropical areas, with the highest prevalence in Central and South America, Africa and Japan [1,3,4]. Sporotrichosis is caused by a complex group of dimorphic fungus species called Sporothrix [1,5-7], the major species includes Sporothrix brasiliensis, S. schenckii, S. globosa and S. albicans and S. mexicana [8-11]. Sporothrix is present on the natural environment in soil, dead wood (Splinter and thorns favor the growth), mosses and cornstalks [12]. But has never been observed as plant pathogen, probably due to the antifungal activity of plants [10].

It grows in the environment in a mold-like form at temperatures between $25-30^{\circ} \mathrm{C}$. Moreover, it develops 


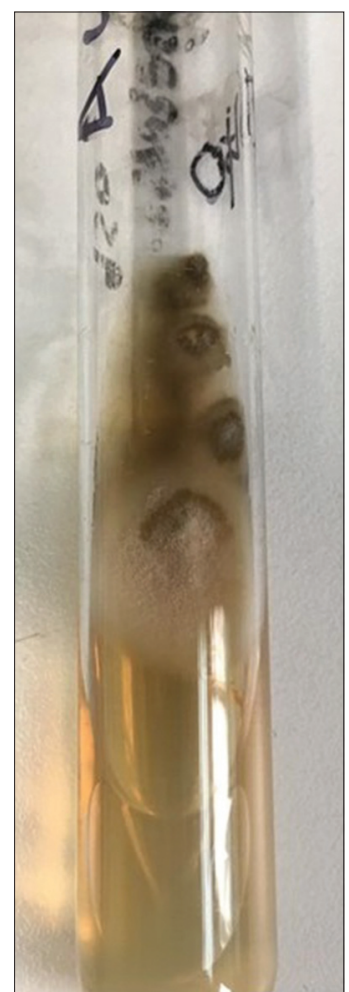

Figure 4: Sabouraud's glucose agar, surface growth is brown, glabrous and yeast like, and reverse is brown.

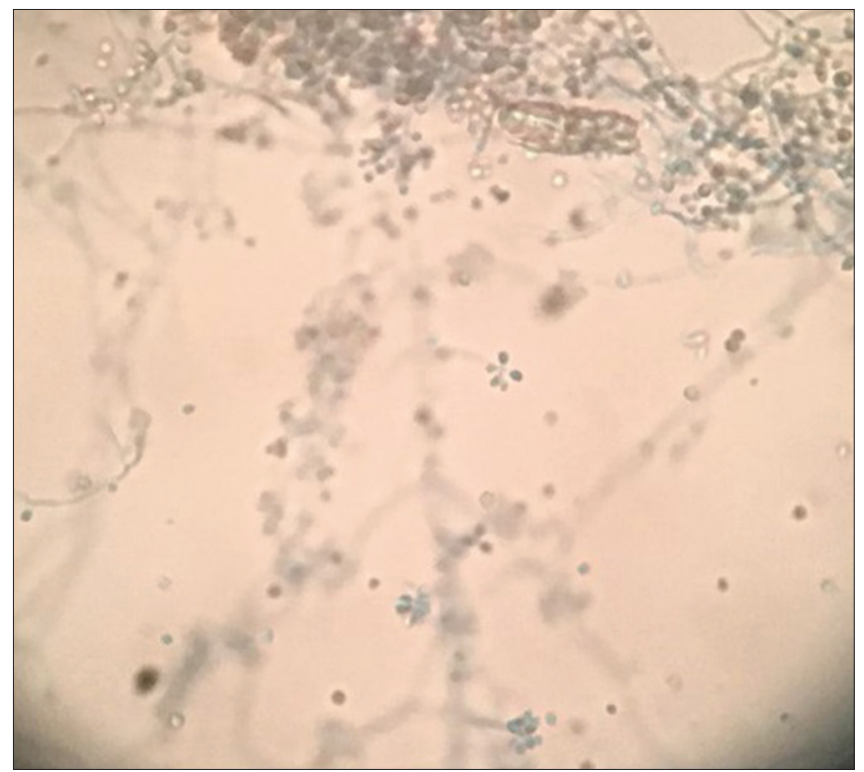

Figure 5: Microscopic morphology of the colony septate and branching hyphae, cluster spores.

into yeast-like form in host tissues at $35-37^{\circ} \mathrm{C}[5,13]$. It is considered an occupational disease of particular risk for agriculturists, gardeners, florists and foresters [2,5]. Furthermore, alcoholism and diabetes have also been correlated as risk factors associated to it [2].

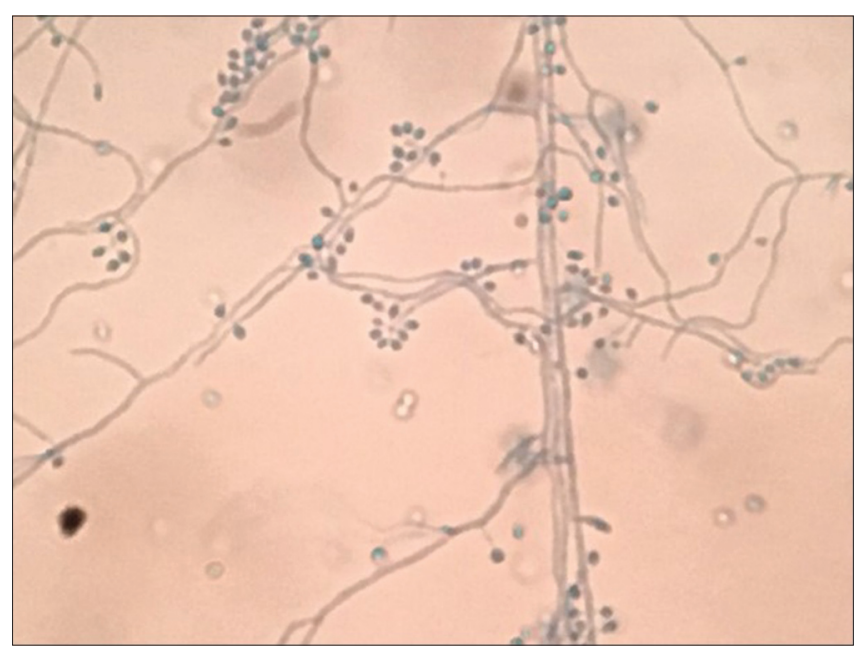

Figure 6: Conidia are in a flowerette arrangement at the single conidiophores.

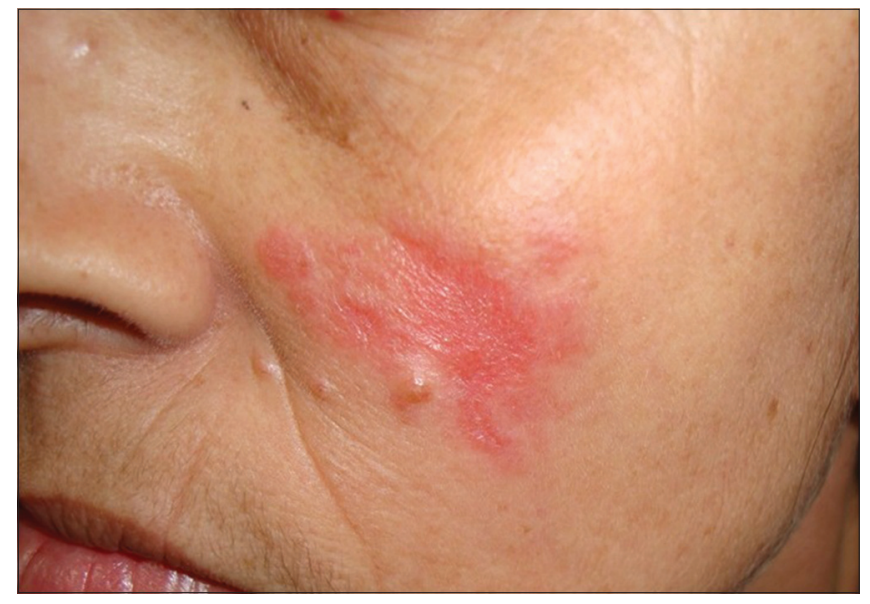

Figure 7: Control post six weeks of treatment with atrophic scar.

This mycosis is more commonly seen in tropical and subtropical countries, principally in Mexico, Central America and Peru. However, the region of Abancay in Perú is considered hyperendemic with an incidence of 48 to 60 cases per 100000 population [11].

This subcutaneous mycosis strongly depends on exposure [12]. It affects individuals regardless of age and gender and it is easily acquired by in children and young adults. Traumatic inoculation is the typical mode for acquisition of cutaneous infection in immunocompetent hosts [5,7], and typically affects the face region and extremities. More recently, it has also been found to be transmitted via domestic cats and rodents in Brazil and Japan [1,8,14].

Several factors such as host immunity, virulence of the inoculated strain and the depth of traumatic inoculation may determinate the different types of presentation [3]. 
Conventionally, three clinical forms are described: 1) Fixed Cutaneous (nodulopapular, ulcerative, verrucouse and furunculoide) $[10,11], 2)$ Lympho-Cutaneous (most commonly) and 3) Disseminated [1,5].

Fixed cutaneous variety of sporotrichosis remains localized and is less common than the lymphocutaneous type $[5,6]$. Clinically, initial lesion develops at the site of skin inoculation [10], commonly on the face region in children and on the arms in adults [14]. This form usually presents a single and painless infiltrated, erythematous or violaceous plaque that may become ulcerated or verrucous without lymphatic involvement $[2,6]$. Also it may start with an acneiform or nodular form of variable duration $[5,14]$.

The lymphocutaneous form is the classical presentation of sporotrichosis and the systemic disease is the product of the conidia inhalation or hematogenous dissemination from primary sites. Pulmonary sporotrichosis displays a form radiographically indistinguishable from tuberculosis and histoplasmosis in patients with sever underlying chronic obstructive pulmonary disease. Osteoarticular sporotrichosis result from the direct inoculation or hematogenous dissemination, with involvement of multiple visceral organs, this occurs almost exclusively in persons with AIDS [10].

Differential diagnoses include cutaneous chronic infections such as cutaneous leshmaniasis in its localized form, but the typical ulcer is painless with erythematous base and well defined high edges [6]. It must be differentiated from tuberculosis, sarcoidosis, paracoccidioidomycosis, chromoblastomycosis, leprosy and other mycobacterial diseases $[2,9]$. Clinical suspicion is essential for diagnosis. Moreover, direct examination of exudate is not suitable due the structures not being observed [2]. The histopathological examination of tissue stained with conventional HE (hematoxylin \& eosin) lacks sensitivity [5]. The gold standard for diagnosis is the isolation of the fungus in Sabouraud dextrose agar with chloramphenicol $[2,11]$.

Prognosis is mainly excellent when proper pharmacological treatment is instituted. Currently, oral itraconazole has become the drug of choice in a recommended dosage of $100-200 \mathrm{mg}$ daily, to be administered for 3-6 months [5]. However, the choice of therapy depends upon the location and form of the disease. Potassium iodide concentrated solution in dosage of 3-6 grams daily for 3-4 months, has been described more affordable and equally effective option in cutaneous forms, especially in endemic areas of developing countries [2,3]. Another therapeutic option is terbinafine, which shows similar results to those of itraconazole [4]. Heat application to lesions may help since low temperatures are preferred by fungus [10].

\section{CONSENT}

The examination of the patient was conducted according to the Declaration of Helsinki principles.

\section{REFERENCES}

1. Larson K, Pandey S, Hoover W, Sun N. Sporotrichosis in the nailAn unusual location and presentation. JAAD Case Rep. 2018;4:47-9.

2. Vásquez-del-Mercado E, Arenas R, Padilla-Desgarenes C. Sporotrichosis. Clin Dermatol. 2012;30:437-43.

3. Franco-Marques G, Prazeres-Sousa J, Wachholz P. Characterization of sporotrichosis cases treated in a dermatologic teaching unit in the State of Sao Paulo - Brazil, 2003-2013. An Bras Dermatol. 2015;90:273-5.

4. Macedo P, Lopes-Bezerra L, Bernardes-Engemann A, OrofinoCosta R. New posology of potassium iodide for the treatment of cutaneous sporotrichosis: study of efficacy and safety in 102 patients. J Europ Acad Dermatol Venereol. 2015;29:719-24.

5. Sudheer N, Venkatakrishna A, Nagasetha M, Monmahan G. Sporotrichosis - A Case Report. J Evol Med Dental Scien. 2015;4:16124-5.

6. Antonio L, Fernandes-Pimentel M, Rosandiski-Lyra M, Madeira M, Campos-Miranda L, Almeida-Paes R, et al. Sporothrix schenckii Sensu Lato identification in fragments of skin lesion cultured in NNN medium for differential diagnosis of cutaneous leishmaniasis. Diag Microbiol Infect Dis. 2017;87:118-20.

7. Lee H, Young-Kim D, Hoon-Lee K, Soo-Choi J, Kyu-Suh M. Deformity of the earlobe caused by fixed cutaneous sporotrichosis in a pediatric patient. Int J Dermatol. 2015;54:187-9.

8. De Araujo M, Rodrigues A, Ferdandes G, De Camargo Z, De Hoog Sybren. Human sporotrichosis beyond the epidemic front reveals classical transmission types in Espírito Santo, Brazil. Mycoses. 2015;58:485-90.

9. Fischman-Gompertz O, Rodrigues A, Fernandes G, Bentubo H, Pires-De-Camargo Z, Petri V. Atypical Clinical Presentation of Sporotrichosis Caused by Sporothrix globosa Resistent to Itraconazole. Am J Trop Med Hyg. 2018;94:1-6.

10. Bimbi C, Brzezinski P. Cutaneous sporotrichosis as an occupational disease: Case report. Our Dermatol Online. 2017;8:37-9.

11. Ramírez Soto MC. Facial Sporotrichosis in children from endemic area in Peru. Our Dermatol Online. 2013;4:237-40.

12. Hakrabarti A, Bonifaz A, Gutierre-Galhardo M, Mochizuki T, Li S. Global epidemiology of sporotrichosis. Med Mycol. 2015;53:3-14.

13. Messias-Rodrigues A, De Hoog S, Zhang Y, Pires-Camargo Z. Emerging sporotrichosis is driven by clonal and recombinant Sporothrix species. Emerg Microbes Infect. 2014;3:1-3.

14. Gyu-Song J, Bum-Song Y, Youl-Yun S, Kyu-Suh M, Yim-Ha G, Ran-Kim J, et al. Cutaneous Sporotrichosis Presenting as Clinical Feature of Facial Cellulitis in an Adult. Ann Dermatol. 2016;28:507-8.

Copyright by Patricia Chang, et al. This is an open access article distributed under the terms of the Creative Commons Attribution License, which permits unrestricted use, distribution, and reproduction in any medium, provided the original author and source are credited.

Source of Support: Nil, Conflict of Interest: None declared. 